\title{
HACIA UN MAPA NARRATOLÓGICO DE LA ‘LEYENDA DE ALEJANDRO MAGNO' EN SU REDACCIÓN SIRIACA
}

\author{
Juan Pedro Monferrer Sala*
}

\section{INTRODUCCIÓN}

El texto siriaco de la 'Leyenda de Alejandro' y el denominado Alexanderlied, narraciones anónimas en ambos casos, representan dos tradiciones textuales distintas en el seno de la literatura siriaca ${ }^{1}$. El texto griego original de la 'Historia' ha sido atribuido tradicionalmente a un autor del periodo helenístico tardío ${ }^{2}$. En realidad, no sabemos a ciencia cierta nada sobre quién fuera el traductor de la versión siriaca del Pseudo Calístenes. Lo más lógico, de acuerdo con la práctica de la producción siriaca de aquellos siglos, es pensar en que el traductor fuese un monje de uno de los muchos monasterios nestorianos que se encontraban en la zona norte de Mesopotamia. La interpolación de elementos cristianos, en ocasiones faltos de precisión, nos llevan a pensar en esta probabilidad ${ }^{3}$.

Está lejos de ser una traducción realizada entre los siglos VII y IX, teniendo como original a un texto árabe, como de facto así lo supusieron Wright y Budge, para quienes la versión siriaca parecía haber sido realizada a partir de una traducción árabe, que a su vez representaría la traducción de una Vorlage griega hecha por un monje cristiano ${ }^{4}$. Sin embargo, la 'Leyenda de Alejandro'

\footnotetext{
* Universidad de Córdoba.
}

1. A. BAUMSTARK, Geschichte der syrischen Literatur mit Ausschluß der christlich-palästinensischen Texte (Bonn, 1922), reed. Berlin, 1968, pp. 125, 191. El mejor estudio hasta el momento es el de Gerrit J. REININK, Das Alexanderlied. Die drei Rezensionen, 2 vols., Louvain, 1983.

2. K. CZEgLÉDY, «The Syriac Legend concerning Alexander the Great», Acta Orientalia, VII, 1957, pp. 231-232.

3. Cf. E.A. WAllis Budge, The history of Alexander the Great, being the Syriac version of the Pseudo Callisthenes (Cambridge, 1889), reed. Piscataway, NJ, 2003, pp. lviii-lx.

4. Ibidem, pp. lx-lxii. 
siriaca es en realidad, una traducción generada por la versión griega del Pseudo-Calístenes a través de una versión neopersa (Pahlawi) de comienzos del siglo VII ${ }^{5}$.

Esta versión parece haber sido obra de un nestoriano ${ }^{6}$ del norte de Mesopotamia y corresponde hacia el año 628. Este texto debió haber sido elaborado poco después de las victoriosas campañas que Heraclio llevó a cabo contra los persas con una finalidad puramente propagandística ${ }^{7}$.

En otro orden de cosas, aunque también ligada a la tradición siriaca, hay que destacar, asimismo, la importancia que tuvieron los textos siriacos en la confección de la tradición árabe de la 'Leyenda de Alejandro', tanto en la tradición cristiana ${ }^{8}$ como en la islámica ${ }^{9}$ y en la judía ${ }^{10}$, así como su ulterior repercusión no sólo en las literaturas orientales, sino también en las occidentales ${ }^{11}$.

Por otro lado, con respecto al célebre memrā que nos ha transmitido una versión de la 'Historia de Alejandro', algunos especialistas han atribuido incorrectamente esta homilía en verso sobre Alejandro Magno al prolífico escri-

5. Th. NÖLDEKE, Beiträge zur Geshichte des Alexanderromans, Vienna, 1890, pp. 11-17. Una reseña del opúsculo de Theodor Nöldeke es la de Siegmund FRAENKEL, «Th. Nöldeke, Beiträge zur Geschichte des Alexanderromans», Zeitschrift der deutschen morgenländischen Gesellschaft, 45:2, 1891, pp. 309-330.

6. Th. NöldeKe, op. cit., p. 17. Cf. A. BAUMSTARK, op. cit., p. 125; K. CZEGlédy, op. cit., pp. 231249; N.V. PIgUleVSKAYA, «Sirijskaya legenda ob Aleksandre Makedonskom», Palestinskii Sbornik, 3, 1958, pp. 75-97. Cf. H. STOKS, «Ein Alexanderbrief in den Acta Cyriaci et Julittae», Zeitschrift für Kirchengeschichte, 31, 1910, pp. 1-47 y P.G. BULGAKOV, «Arabskaya parallel k 24 glave III knigi sirijskoj versii romana ob Aleksandre», Palestinskii Sbornik, 2, 1956, pp. 53-57.

7. G.J. REININK, «Die Entstehung der syrischen Alexanderlegende als politisch-religiöse Propagandaschrift für Herakleios' Kirchenpolitik», en C. LAGA, J.A. MunITIZ y L. VAN ROMPAY (eds.), After Chalcedon. Studies in Theology and Church History, offered to Professor Albert van Roey for his seventieth birthday, Louvain, 1985, pp. 263-281. Cf. S.P. BROCK, A brief outline of Syriac literature, Kotayyam, India, 1997, p. 51.

8. S.Kh. SAMIR, «Les versions arabes chrétiennes du Roman d'Alexandre», en Rosa Bianca FINAZZI y Alfredo VALVO (eds.), La difussione dell'eredità classica nell'età tardoantica e medievale. Il "Romanzo di Alessandro" e altri scritti, Alessandria, 1998, pp. 227-247. Cf. Stephen GERÖ, «The legend of Alexander the Great in the Christian Orient», Bulletin of the John Rylands University Library Of Manchester, 75:1, 1993, pp. 3-9.

9. M.M. MazZAouI, «Alexander the Great and the Arab historians», Graeco-Arabica, 4, 1991, pp. 33-43; M. MARíN, «Legends on Alexander the Great and the Arab historians», Graeco-Arabica, 4, 1991, pp. 71-89; V. PogGI, «Alessandro Magno, dal 'Romanzo' alla sura 'della caverna'», en Rosa Bianca FINAZZI y Alfredo VALVO (eds.), La difussione dell'eredità classica nell'età tardoantica e medievale, pp. 197-208; Z.D. ZuWIYYA, Islamic Legends Concerning Alexander the Great Taken from Two Medieval Arabic Manuscripts in Madrid, Introduction, edition, translation, and notes by Z. David Zuwiyya, Binghampton, NY, 2001.

10. F. Pfister, Alexander der Grosse in den Offenbarungen der Griechen, Juden und Mohammedaner und Christen, Berlin, 1956.

11. J.A. BoYLE, «The Alexander Romance in the East and West», Bulletin of the John Rylands University Library of Manchester, 60:1, 1977, pp. 13-27. 
tor Jacobo de Sĕrūg ${ }^{12}$, tal como consta en los MSS siriacos que han llegado hasta nosotros, la cual, reelaborada ulteriormente, según algunos, habría dado lugar a las versiones amplificadas posteriores que conocemos ${ }^{13}$.

Así pues, mientras que por el momento nada sabemos sobre quién pueda ser el autor de la traducción de la 'Leyenda de Alejandro', así como tampoco el de la recensión de aquélla, conocida como 'Proezas de Alejandro", sí que tenemos noticias, por el contrario, del autor eclesiástico al que se le atribuye el memrā de la 'Historia de Alejandro': Jacobo de Sĕrūg. Se trata de un obispo monofisita de la localidad de Sĕrūg, que murió en el año 521. De este personaje poseemos tres biografías: la escrita por Jacobo de Edesa ${ }^{14}$, una segunda anónima y la tercera, que unos atribuyen a un discípulo suyo llamado Jorge, mientras que otros se la atribuyen a un escritor del s. VIII llamado Jorge de Sĕrūg $(\uparrow 708)^{15}$.

Nacido en Kurtam, una población situada sobre el río Éufrates, Jacobo de Sĕrūg fue educado en la célebre 'Escuela Persa' de Edesa contra cuyos postulados cristológicos reaccionó, siendo ordenado corepíscopo en la región de Sĕrūg, concretamente en Hawrā, al suroeste de Edesa, y más tarde, en el año 519, fue ordenado obispo en Bațnān d-Sĕrūg. La fama de este autor reside, esencialmente, en el ingente número de memrē que compuso sobre distintos tópicos, sirviéndose para ello del metro dodecasilábico, en algunos de los cuales se aprecia la influencia de la exégesis de Teodoro de Mompsuestia aprendida en la 'Escuela Persa' de Edesa. Compuso, además, 6 homilías en prosa (turgame ), 43 epístolas (entre las que destacan aquellas remitidas a los cristianos de Nağrān), varias odas (madrāšse) e himnos (sugyātēe), así como el servicio bautismal maronita, que también le ha sido atribuido ${ }^{16}$.

12. Carl Hunnius, Das syrische Alexanderlied, Götingen, 1904, Inaugural-Dissertation; E. NESTLE, «Zum syrischen Alexanderlied in Heft I», Zeitschrift der deutschen morgenländischen Gesellschaft, 50, 1906, pp. 401-402; E.A. WALLIS BuDGE, «The Homily on Alexander the Great attributed to James, Bishop of Bațnān», Zeitschrift für Assyriologie, 6, 1891, pp. 357-404; A.R. ANDERSON, Alexander's Gate. Gog and Magog and the Enclosed Nations, Cambridge, Mass, 1932; K. CzEGLÉDY, «Monographs on Syriac and Muhammadan sources in the literary remains of M. Kmosko», Acta Orientalia, 4, 1955, pp. 19-90, y K. CZEGLÉDY, «The Syriac Legend...», pp. 231-249.

13. C. HunNIUS, «Das syrische Alexanderlied», Zeitschrift der deutschen morgenländischen Gesellschaft, 60, 1906, pp. 170-171. Cf. R. Duval, La littérature syriaque des origines jusqu'à la fin de cette littérature après la conquête par les arabes au XIIle siècle (Paris, 1907), reed. Amsterdam, 1970, pp. 321-322; A. BAUMSTARK, Die christlichen Literaturen des Orients. I. Das christlich-aramäische und das koptische Schrifttum, Leipzig, 1911, p. 103; C. BROCKELMANN, «Die syrische und die christlicharabische Literatur», en C. BROCKELMANN, F.N. FINK, J. LEIPOLDT y E. LITTMANN, Geschichte der christlichen Literaturen des Orients (Leipzig, 1909), reed. Leipzig, 1979, p. 26, n. 1; K. CZEGLÉDY, «Monographs on Syriac and Muhammadan sources...», p. 35.

14. J.S. Assemanus, Bibliotheca Orientalis Clementino-Vaticana. I. Sriptoribus Syris Orthodoxis (Rome, 1719), reed. Piscataway, NJ, 2002, p. 286.

15. R. DUVAL, op. cit., p. 352.

16. A. BAUMSTARK, Geschichte der syrischen Literatur.., pp. 148-158. Cf. R. DuvAL, op. cit., pp. 352-354; W. WRIGHT, A short history of Syriac literature, London, 1894, pp. 67-72; W. STEWART MCCULlOUGH, A short history of Syriac Christianity to the rise of Islam, Chico, CA, 1982, p. 79; S.P. BROCK, op. cit., pp. 36-37. 


\section{ESTRUCTURA Y ARGUMENTACIÓN TEMÁTICAS}

La estructura del texto siriaco de la 'Leyenda de Alejandro' está claramente descompensada en las tres secciones en las que aparece dividida la obra. El rasgo principal, a primera vista, es la rica variedad temática que presenta el texto ${ }^{17}$, variedad que se sucede por medio de las sagas que componen la obra, con las que el autor logró confeccionar un producto narrativo de carácter pluritemático. No podemos hablar, por lo tanto, de un único tema. Obviamente, todos los acontecimientos expuestos y descritos a lo largo del texto evolucionan en función de la figura del personaje central, Alejandro.

Este personaje, así pues, es el dinamizador de cada uno de los ciclos temáticos que genera su leyenda. A él se refiere cada acción concreta del texto y sólo a través de él tiene sentido cada desarrollo incluido en la obra. Las mismas secuencias de tipología descriptiva ( $v$.gr. la denominada "saga de las amazonas") o argumentativa (v.gr. "la carta a Aristóteles") $)^{18}$, entre otras claramente perceptibles en la estructura de la obra, no son sino digresiones que cumplen con la función narratológica de replantear la trama narrativa de la obra introduciendo las nuevas sagas, lo que, a su vez, hace que se sucedan los diversos ciclos narrativos que integran la obra.

Podemos avanzar que son, sustancialmente, tres las horæ narrativæ que marcan el ductus temático del conjunto del texto, tal como señalamos a continuación:

1. Juventud y aprendizaje de Alejandro.

2. Empresa militar de Alejandro contra macedonios y griegos, persas e indios.

3. Testamento y muerte de Alejandro.

Estos tres elementos temáticos, como puede apreciarse en la estructura de la obra a la que acabamos de referirnos, se encuentran narrativamente unidos mediante una serie de secuencias narrativas que funcionan como transiciones. A éstas, de suyo, las podemos calificar como elementos subtemáticos que pertenecen a diversas tipologías narrativas: a) desde digresiones hasta b) textos epistolares, pasando por c) fragmentos descriptivos. De acuerdo con lo que acabamos de exponer, una estructuración sintética de los contenidos que presenta la 'Leyenda de Alejandro' puede quedar del modo que exponemos a continuación.

17. Cl.A. CiAnCAGLINI, «Gli antecedenti del Romanzo siriaco di Alessandro», en Rosa Bianca FinAZZI y Alfredo VAlvo (eds.), La difussione dell'eredità classica nell'età tardoantica e medievale, pp. 55-93. Cf. R. JASNOW, «The Greek Alexander Romance and Demotic Egyptian Literature», Journal of the Near East Studies, 56:2, 1997, pp. 95-103 y R. MERKELBACH, Die Quellen der griechischen Alexanderromans, München, $1977=1954$.

18. Sobre la importancia de ésta en la literatura árabe, véase J. BIELAWSKI, «Lettres d'Aristote à Alexandre le Grand (et lettres d'Alexandre à Aristote) en version arabe», Rivista di Studi Orientali, 28,1964 , pp. 7-34 y 29, 1965, pp. 7-12 
El monarca-mago egipcio Nectanebo marcha a Macedonia debido a una traición que los dioses han urdido y traba contacto con Felipe y Olimpia. Haciéndose pasar por Ammón, el dios de Libia, Nectanebo hará que Olimpia conciba un hijo, al que Felipe, su padre adoptivo, dará por nombre Alejandro.

Educado por los mejores instructores, el joven Alejandro pronto debe hacer frente a diversas empresas políticas y militares, además de mostrar su capacidad en una competición tan emblemática para un héroe de la antigüedad como lo eran las carreras de caballos.

La perfecta instrucción recibida por Alejandro, en combinación con la propia coyuntura de la situación geopolítica que se vive en esos momentos, lleva a éste a tener que afrontar una situación incomparable: a saber, crear un imperio que no tendrá par en la historia. De este modo, el nudo central de la obra está narrativamente constituido por la exposición del control de las ciudades y de los territorios macedonio y griego, la consolidación de las instituciones político-religiosas (muy sucintamente tratadas en el texto, pero que son evidentes en la obra) y la ocupación y la administración del imperio persa ${ }^{19}$, unificado por Darío. Todos los subtemas que forman parte de cada uno de estos ciclos se encuentran articulados por medio del tema bélico de la conquista-expansión que lleva a Alejandro a levantar su imponente imperio.

Por su parte, el colofón narrativo de la historia viene dado por una modalidad narrativa que experimentó un creciente desarrollo en la tardoantigüedad, los denominados "discursos del adiós". La profecía que vaticina la muerte de Alejandro abre este ciclo por medio del propio vaticinium ex eventu y la conspiración que se urde con ella para intentar así acabar con Alejandro ${ }^{20}$. La sección central de este ciclo de la muerte de Alejandro está formada por el testamento y, a su vez, la conclusión de la obra por la muerte de Alejandro, que acaba siendo enterrado en la gran ciudad que fundara y recibiera su propio nombre, Alejandría.

Esta pluralidad de contenidos o subtemas parecen estructurarse en torno a un concepto escatológico de gran importancia en el medio siriaco del siglo VII, que en buena medida condiciona y articula la producción literaria del momento. Así, por ejemplo, en este marco escatológico de tipología apocalíptica la 'Leyenda de Alejandro' se convierte en una de las fuentes principales de los textos apocalípticos entre los que sobresale el célebre 'Apocalipsis del Pseudo Metodio ${ }^{21}$.

19. A. SHApUR SHAhbAZI, «Iranians and Alexander», American Journal of Ancient History, 2, 2003, pp. 5-38.

20. Sobre el desarrollo del motivo de la muerte de Alejandro en las distintas versiones medievales, véanse L. DepuydT, «The Time of Death of Alexander the Great», Welt des Orients, 28, 1997, pp. 117-135 y los diez artículos incluidos en W.J. AERTS, Jos. M.M. Hermans y Elizabeth VISSER (eds.), Alexander the Great in the Middle Ages. Ten Studies on the Last Days of Alexander in Literary and Historical Writing, Symposium Interfacultaire Werkgroep Mediaevistiek (Groningen 12-15 October, 1977), Nijmegen, 1978.

21. Gerrit J. REININK, «'Rede über das Ende' und die syrische eschatologische Literatur des siebenten Jahrhunderts», Aram, 5, 1993, pp. 437-463; Idem, «Alexander the Great in SeventhCentury Syriac 'Apocalyptic' Texts», Byzantinorossica, 2, 2003, pp. 150-178. Cf. F.J. MARTínez, 
La 'Leyenda de Alejandro', como también sucede con otras obras siriacas pertenecientes al género apocalíptico, pero sobre todo en la recensión de aquélla, conocida como 'Proezas de Alejandro', a la que nos hemos referido anteriormente, tiene un importante contenido programático de tipología propagandística con la que reivindicar el prestigio del basileo bizantino Heraclio como vencedor sobre el ejército persa sasánida ${ }^{22}$, recurriendo para ello a la identificación entre Heraclio y su arquetipo Alejandro. Alejandro, de este modo, representa el paradigma del emperador, que para el autor siriaco cristiano, evidentemente, es el del emperador cristiano cuya actuación terrena se conduce por la voluntad divina. Alejandro, tal como sucede en el memrā atribuido a Jacobo de Sĕrūg, representa al emperador pío al que se le encomienda la misión divina de fundar un imperio universal que se conduzca por medio de la ley divina ${ }^{23}$.

De acuerdo con todo ello, la variada temática que conforma el texto siriaco de la 'Leyenda de Alejandro' representa un tipo de Historia salutis en el que los acontecimientos que se van sucediendo por medio de la compleja trama urdida por el autor, ya sean de naturaleza política, bélica o moral, obedecen en realidad a acciones desencadenadas por la divinidad. En el texto de la 'Leyenda de Alejandro' el elemento divino está representado por Nectanebo, en tanto que en la cristianizada "Proezas de Alejandro" esta divinidad no es otra que el Dios cristiano.

Estamos, por lo tanto, en el caso concreto de la 'Leyenda de Alejandro' siriaca, ante una visión del concepto de historia que no es en modo alguno meramente político, sino más bien soteriológico, utópico por lo tanto. La 'Leyenda de Alejandro' se abre y se cierra con Alejandro como modelo a seguir, a imitar, como un rey creyente que hace cumplir la voluntad divina en la tierra. Todo este marco soteriológico alcanza su clímax en el 'Testamento' (v.gr. cap. III, 22). En este capítulo, a imagen de Cristo, cuando éste envía a sus seguidores a difundir la buena nueva por todo el mundo (Mt 28,19 par.), del mismo modo Alejandro reparte su imperio entre sus colaboradores para que así éstos puedan continuar la labor iniciada por él.

La figura de Alejandro, por lo tanto, siendo un héroe no cristiano se convierte en elemento susceptible de convertirse en modelo de los textos de género legendario cristiano ${ }^{24}$, que el formato de la recensión adaptó de modo inteligente en el seno de la tradición siriaca. En este proceso, la traducción de la 'Leyenda de Alejandro' desempeñó, sin duda, un valor de primer orden.

Eastern Christian Apocalyptic in the Early Muslim Period: Pseudo-Methodius and Pseudo-Athanasius, Ann Arbor, Mi., 1996, pp. 18-19; también A. PALMER, The Seventh Century in the West-Syrian Chronicles, pp. 223, 239.

22. G.J. REININK, «Die Entstehung der syrischen Alexanderlegende...», pp. 263-281.

23. G.J. REININK, «Alexandre et le dernier empereur du monde: les développements du concept de la royauté chrétienne dans les sources syriaques deu septième siècle», en Laurence HARF-LANCNER, Claire KAPPLER y François SUARD (eds.), Alexandre le Grand dans les littératures occidentales et proche-orientales. Actes du Colloquie de Paris (27-29 novembre, 1999), Nanterre, 1999, pp. 152-154.

24. Cf. A. Abel, «Dū l-Qarnayn, Prophète de l'universalité», Annuaire de l'Institut de Philologie et d'Histoire Orientales et Slaves, 11, 1951, pp. 5-18. 


\section{TÉCNICA Y ESTILO NARRATIVOS}

También la técnica narrativa y el estilo utilizados en la 'Leyenda de Alejandro' dejaron su impronta en la versión siriaca. En primer lugar debemos precisar que la 'Leyenda de Alejandro' siriaca, por medio de la traducción que es, representa el producto final de una doble encrucijada textual: un texto griego original, a través de la mediación de una versión neopersa ${ }^{25}$. De este modo, la convención original adoptada en la recensión $\delta$ del Pseudo Calístenes griego se verá modelada por las variantes genuinas propias de la versión neopersa, que, de este modo, redefinirá aquellos elementos textuales que son tenidos por básicos y dan el carácter constitutivo de un texto narrativo: "originalidad", "convención" e "influencia".

Esta "arqueología del texto" es un elemento que desempeña un papel de enorme relevancia a lo largo de la obra, puesto que la pluralidad estructural que subyace a un texto, que representa a su vez una versión de otro texto anterior en una lengua distinta, es producto de una labor interpretativa de la Vorlage. Tales características exigen que el traductor realice una labor de imitatio que, ineludiblemente, llevará a éste a regenerar el texto en no pocos loca narrationis.

En no escasa medida, toda traducción, aunque sea por medio de elementos insignificantes, realiza una tarea de adaptación con la que el traductor contribuye, aunque sea de modo marginal, a la temática de la obra. En este sentido, un rasgo característico de la versión siriaca son las lecturas erróneas que presenta la realización de los nombres propios, que sólo pueden conducir a un texto neopersa. Por otro lado, el uso específico de neologismos y de calcos semánticos es asimismo un producto resultante de la traducción, no de una obra original. De este modo, muchos son los elementos que el narrador va introduciendo, amalgamando, amplificando o elidiendo, según sea el caso, a lo largo del texto: sucesión de cuadros descriptivos y monológicos, genuinamente epistolográficos, así como secuencias dialógicas que presentan una atmósfera narrativa propicia para un variado uso de elementos figurativos y tropológicos.

Por otro lado, en lo tocante al marco superestructural de los géneros, al igual que acontece en tantas obras de la tardoantigüedad, aunque no exclusivamente, la 'Leyenda de Alejandro' no conoce la división aristotélica que se estableció entre literatura de ficción y literatura didáctica, ya que las fronteras de los géneros literarios aún no estaban definidas en esos momentos. Aunque se trata de una obra estructuralmente iterativa, las variaciones temáticas imprimen una dinámica narrativa concreta, aunque falta, sin embargo, una capacidad espaciadora a nivel argumental en la transición entre los diferentes cuadros narrativos. Además, la propia linealidad que presenta el relato carece de una

25. Sobre la recepción irania de Alejandro, véase por ejemplo A. ABEL, «La figure d’Alexandre en Iran», en La Persia e il mondo greco-romano, Roma, 1966, pp. 119-134. 
cronología narrativa rigurosa. Esto provoca que la obra se inserte en un espacio textual atemporal, que obviamente neutraliza espacios y tiempos históricos.

La obra, mediante el recurso del modelo narrativo historiográfico-legendario, contempla dos planos de significación: uno moral y otro histórico. Ambos, ejemplificados por medio de las actuaciones de Alejandro, persiguen proyectar una nueva dimensión histórica que es dual: la del helenismo como nuevo marco histórico geográfico-político-cultural y la del nuevo modelo de monarca como personaje supradimensional que aúna la perfección ética y moral.

Esta nueva dimensión histórica, lógicamente, supone una progresiva ruptura con el modelo anterior: del reducido ámbito de la polis se pasa a un marco colonizador en todas sus posibilidades: geográfica, política, social, religiosa y cultural, que el narrador va describiendo de modo admirable y progresivo. No sólo cambia el marco geográfico, sino que también cambia el registro político, que, en hábil combinación narrativa con los elementos restantes, consigue conformar la denominada "cultura espiritual del helenismo".

Desde el punto de vista geográfico, aunque este recurso narrativo no responda a una localización topográfica precisa en todos sus elementos ${ }^{26}$, Oriente representa el marco y su contexto, pero el punto neurálgico e ideológico es Alejandría, que representa el modelo supradimensional de los nuevos arquetipos: grandes construcciones como modelo de un gigantesco imperio hecho a imagen de su emperador.

El objetivo básico de la obra es esencialmente didáctico y, por ende, instructivo y formativo. Sin embargo, este concepto no debemos entenderlo desde el punto de vista genérico, sino como mero elemento articulatorio a nivel tipológico. La exposición narrativa, partiendo del criterio básico de la praxis historiográfica, ha adoptado el modelo del tipo historiográfico novelado con el objetivo claro de obtener una historia dramatizada. De este modo, la "fidelidad histórica", como elemento activador de una teleología historiográfica que ha planteado la narratio, se encuentra en todo momento al servicio del drama histórico que vive la historia a través de Alexander.

\section{PERSONA NARRATIONIS}

Una importancia temática y estructural obvia desempeñan los principales personajes que actúan en los diversos episodios de la 'Leyenda de Alejandro', hasta erigirse, de hecho, en parte constitutiva de un microcosmos. Estos personajes han sido confeccionados a base de topoi que amalgaman rasgos diversos: históricos, geográficos, culturales, etc., pero con los que, al propio tiem-

26. A.R. ANDERSON, «Alexander at the Caspian Gates», Transactions and Proceedings of the American Philological Association, 59, 1928, pp. 130-163; F. PFISTER, «Studien zur Sagengeographie», Symbolae Osloenses, 35, 1959, pp. 22-28. 
po, el narrador configura el cañamazo estructural planificado ad hoc por éste. Toda esta elaboración forma parte de una tradición historiográfico-narrativa que remonta a la época helenística, con confluencias con el género de los relatos autobiográficos y epistolográficos de los héroes por medio de los recursos monológico y dialógico. Obviamente, esta tradición clásica recibe interferencias puntuales con respecto a los personajes por medio de la técnica de la contaminatio, como sucede en el episodio que describe el momento de la muerte del rey persa Darío, que es el resultado de una clara interpolación cristiana.

El producto narrativo generado por el narrador en materia de caracterización de personajes es realmente interesante. En efecto, nos encontramos ante un espléndido observador, y lo que es más, ante un excelente fabricante de arquetipos humanos. La dialéctica que abre entre los personajes que crea el narrador a base de polarizar las dimensiones morales genera todo un proceso de simbolización que define y marca los rasgos de los caracteres orales y éticos de los personajes, así como su consiguiente desarrollo y final narrativo. Mientras que Alejandro es tratado como un personaje mítico que reúne en su persona toda perfección posible, la valoración de los personajes restantes responde, por el contrario, a una gradación variable establecida en función del nivel al que pertenece cada uno de ellos, pero eso sí respondiendo en todo momento a arquetipos humanos que siempre se encuentran por debajo de la figura de Alejandro. De todo lo anterior se deduce, consiguientemente, toda una clasificación general de los personajes principales obtenida a partir de la función primaria que desempeñan en la obra con obvias aplicaciones de naturaleza estructural:

A) Principales:

a) Partidarios: Nectanebo (desdoblado en Ammón, Heracles, Dionisos), Olimpia, Felipe.

b) Opositores: Darío, Poro, Qerātōr, Antípatro, Yolas, Casandra.

B) Secundarios:

a) Transicionales: Pitia, Zintōs, Nicolás, Cleopatra, Lísias, Teósido, Guštāzaf, Sābānțār, Clitómaco, Asquino, Demades, Demóstenes, Pasargēs, Bāgīz, Ānābdēh, Gundāfār, Paryōg, Prīsqōs, Ptolomeo.

b) Autoritativos: Aristóteles.

c) Disgresivos: el grupo de los Brahmanes, las Amazonas, Candaces.

d) Proféticos: Antífono el augur, la mujer con el monstruo de las cuatro cabezas (cap. III,19).

e) Residuales: Rōšnāq, Yirāndūknt, 'Esțehar (la hija, la madre y la esposa de Darío respectivamente). 


\section{EL NARRADOR Y SUS INSTRUMENTA NARRATIONIS}

En la 'Leyenda de Alejandro' más que hablar de un narrador propiamente dicho habría que referirse, de modo más acertado, a esa modalidad discursiva que conocemos técnicamente como narratio. Esta técnica, elemento esencial en la elaboración de la materia discursiva, se encarga, propiamente, de la exposición y de la disposición del material. La narratio, de hecho, se amolda constantemente a lo largo de toda la obra a cualquier tipo de molde narratológico: digresiones de naturaleza histórica, proemios o exordios de una determinada saga, descripciones de acontecimientos varios, etc.

En el caso concreto de la versión siriaca de la 'Leyenda de Alejandro', por lo tanto, la figura del narrador es ocupada en muchos momentos por la narratio propiamente dicha. En este sentido, el método narratológico empleado por el narrador resulta aparentemente simple si lo comparamos con las técnicas narrativas utilizadas en textos posteriores. Ahora bien, ésta sólo lo es comparativamente hablando, porque el procedimiento seguido por su autor, no siendo novedoso en ninguno de sus aspectos, es el arquetipo del modelo helenístico que pervivirá posteriormente en otras tradiciones literarias siriacas que son sin duda deudoras de aquella ${ }^{27}$, como es el caso de no pocas muestras de la literatura siriaca.

Los rasgos estilísticos más sobresalientes de la obra son fruto del marco trágico que planea sobre la escena en todo momento. En este marco, la figura del paralelismo desempeña una función constante entre los diversos episodios que se suceden de principio a fin de la 'Leyenda de Alejandro'. Este procedimiento, de hecho, es el que determina la estructura de la obra, estableciendo de este modo una especie de subordinación narrativa lógica en el texto, con el que el narrador le confiere una disposición simétrica. El paralelismo se convierte, en este sentido, en una metáfora espacial, dado que configura un diseño estructural muy preciso en el que la linealidad de lo narrado aparece dispuesta mediante un proceso de acciones temáticas paralelas que se suceden espacialmente unas a otras.

De principio a fin, la 'Leyenda de Alejandro' engarza una sucesión continua de episodios en los que la historiografía y la biografía se (con)funden y se yuxtaponen. Por ello, el rasgo estilístico más común es el de la descripción con la que son presentados los objetos, los seres y los lugares, de los que se nos explica sus partes, así como sus cualidades y hasta se nos indica las circunstancias en las que se encuentran éstos envueltos, como si el narrador describiese con gran viveza figurativa elementos que ha presenciado previamente con sus mismos ojos.

27. L.I. CONRAD, «Varietas Syriaca: Secular and Scientific Culture in the Christian Communities of Syria after the Arab Conquest», en G.J. ReInINK y A.C. KLugkist (eds.), After Bardaisan: Studies on Continuity and Change in Syriac Christianity in Honour of Professor Han J. W. Drijvers, Leuven, 1999, pp. 85-105. 
La descripción es completada con otros elementos descriptivos como la prosopografía, gracias a la cual el narrador ofrece logrados cuadros descriptivos externos de esas mismas categorías. La etopeya, por su parte, aunque menos frecuente, acompaña las descripciones de algunos personajes para describir las cualidades morales de éstos. Fruto de la fusión de estos elementos descriptivos previos, prosopografía y etopeya, en un único elemento es el retrato, ya parcial ya completo, gracias al cual obtenemos perfiles físicos y físicosmorales de un personaje.

Otro elemento descriptivo de gran repercusión en la obra es el de la topografía, utilizado por el narrador para describir lugares. A éste, además, se suman rasgos procedentes de la historiografía puramente factual con los que el narrador ofrece datos fruto de una realidad que conforma el substrato histórico del relato. Así, tropos descriptivos como la enumeración rápida y breve de objetos o de ideas son habituales a lo largo del texto. La función de estos tropos no es otra que la de destacar los objetos enumerados con una visión que va de lo cuantificador a lo cualificador, siempre dentro de un interés realista de corte historiográfico.

El patetismo de la obra es evidente en las figuras que describen la emoción, la fuerza que experimenta el narrador en determinados puntos del texto. En este sentido, los apóstrofes o invocaciones vocativas suelen interrumpir el desarrollo narrativo para enfatizar un elemento o un personaje e incluso para aislar de un punto narrativo determinado al personaje que habla. Menos habituales, aunque también presentes en la obra, son otras figuras patéticas: por ejemplo, las antitéticas de la deprecación y la imprecación, la maldición, la execración o la conminación que aparecen en el texto.

Tropos pertenecientes al nivel léxico-semántico con una función gradadora del objeto descrito son la hipérbole y la prosopopeya, presentes en los cuadros narrativos que podríamos clasificar como supra-naturales. Asimismo, el narrador se sirve de figuras lógicas como la sentencia en forma de máxima que en ocasiones se transforma en un epifonema. Habitual es el símil, cuya finalidad explicativa consigue gradaciones descriptivas de valor, gracias a las cuales se consigue un clímax ascendente que confiere al texto un dinamismo narrativo. Por su parte, el uso de la perífrasis provoca, en determinadas ocasiones, una ralentización que permite al narrador detenerse en determinados cuadros narrativos.

Tropos en forma de sinécdoque o de metonimia, metáfora, alegoría, profecía o símbolos cumplen funciones que van desde la compleción de una enumeración hasta una proyección del sentido o a la concreción de abstracciones, la formulación de un futurible o la simbolización de determinados elementos o ideas. En este sentido, metáforas como "mar" o "tierra", por ejemplo, desempeñan un papel determinante de carácter dinámico en los continuos movimientos de Alejandro. Ambas metáforas puras se convierten, de este modo, en arquetipos de la dinamicidad de la vida de éste, pero siendo entendidas, sobre 
todo, como trayecto hacia un cambio de modelo: de la polis al imperio, del jefe o régulo al emperador. El mar, además, posee un sentido bivalente: es, al mismo tiempo, el lugar de la muerte y el de la vuelta a la vida. El mar y la tierra, de este modo, constantemente surcadas por Alejandro se convierten en elementos argumentales claves que marcan un punto sin retorno en la historia. Es el continuo avanzar de la narración, de la "historia", hacia las sucesivas anagnórisis finales.

\section{A MODO DE CONCLUSIÓN}

El narrador de la 'Leyenda de Alejandro' no se interesa por la historia en sí misma, sino como medio con el que llevar adelante su propósito de modelizar la figura de Alejandro en tanto que héroe en toda su dimensión legendaria y arquetípica al mismo tiempo: en los planos humano, político, bélico, etc. Es ese el motivo por el que el narrador realiza una cuidada selección de los segmentos narrativos que mejor puedan contribuir a modelar el arquetipo de su personaje, que le ayuden a proyectar al Alejandro histórico y al Alejandro legendario a un mismo tiempo.

Como el material narrativo no siempre resulta temáticamente traslúcido para el lector, el narrador se siente en la necesidad de reflejar su intencionalidad en esta selección del material, así como en la (re-)elaboración y (re-)interpretación de los datos historiográficos fijados a partir de topoi procedentes, en cada caso concreto, de la tradición historiográfica helenística o, en su defecto, persa.

En este sentido, las digresiones explicativas, los juicios de valor como aportación personal o la argumentación discursiva de tipología interpretativa de las que se sirve el narrador se erigen en los instrumenta, mejor aún en la base constitutiva de su modelo narratológico. De hecho, el narrador maneja a la perfección la presentación de la niñez de Alejandro, valiéndose para ello de asuntos y temas genuinos del marco socio-histórico propio de las fuentes utilizadas en la composición de la obra. A los primeros años formativos del personaje sucede la actuación política y posteriormente sus logros bélicos, para lo cual utiliza conocidos topoi de tipología narrativa biográfica.

Esta triple dimensión orgánica ha sido narratológicamente homogeneizada por el narrador mediante una argumentación subliminal con la que obtiene el marco humano del personaje, el de la dimensión ético-moral de Alejandro como arquetipo histórico, que condiciona no sólo el devenir temático de la obra, sino la propia labor compositiva que lleva a cabo el narrador. En no pocos aspectos, la lectura modélica que propone el narrador persigue, en todo momento, realizar una (re)construcción de los cánones sociales y culturales de la época, pues para los lectores contemporáneos del autor la obra reflejaba, sin duda alguna, unos contenidos que eran auténticamente veraces y por lo tanto históricos. 


\title{
RESUMEN
}

Análisis de la Leyenda de Alejandro Magno en su versión siriaca, comparándola con otros textos siriacos paralelos y situándola cronológica y cotextualmente en su ámbito de creación y difusión. Indicación de las huellas que pueda tener de versiones posteriores en otras lenguas, con preferencia de una versión en neopersa. Estudio de la construcción de la figura de Alejandro como héroe arquetípico ejemplar a través de un cuidado trabajo literario a todos los niveles, que llevan a considerar la Leyenda de Alejandro Magno como un texto bien trabado que, a través de la reconstrucción de los cánones sociales y culturales de la época de su composición, se adecua al público al que iba dirigido.

Palabras clave: Leyenda de Alejandro Magno, siriaco, ejemplaridad, arquetipo.

\begin{abstract}
Analysis of the "Legend of Alexander the Great" in its Syriac version, compared to other parallel Syriac texts, and framing it in its chronological and co-textual creation and dissemination context. Determination of the traces of later versions to other languages, with preference to a neo-Persian version. Study of the construction of the figure of Alexander as an archetypical and exemplary hero, through a careful literary study at all levels, which lead to consider the "Legend of Alexander the Great" as a well-constructed text, which is adapted to its intended audience by means of a reconstruction of the social and cultural canons of the period.
\end{abstract}

Key words: Legend of Alexander the Great, Syriac, exemplarity, archetype. 